\title{
A Low-cost Zn-based Aqueous Supercapacitor with High Energy Density
}

Liang $\mathrm{He}^{\mathrm{a} \#}, \mathrm{Yu} \mathrm{Liu}^{\mathrm{a} \#}$, Chunyang $\mathrm{Li}^{\mathrm{a}}$, Dezhi Yanga ${ }^{\mathrm{a}}$, Weigang Wanga ${ }^{\mathrm{a}}$, Wenqi Yan ${ }^{\mathrm{a}}$, Weibin Zhou ${ }^{\mathrm{a}}$, Zhixian Wu $\mathrm{W}^{\mathrm{a}}$, Lili Wang ${ }^{\mathrm{a}}$, Qinghong Huang ${ }^{\mathrm{a}}$, Yusong Zhu ${ }^{\mathrm{a}}$, Yuhui Chen ${ }^{\mathrm{a}}$, Lijun $\mathrm{Fu}^{\mathrm{a}, \mathrm{b} *}$, Xianhua $\mathrm{Hou}^{\mathrm{b} *}$ and Yuping $\mathrm{Wu}^{\mathrm{a}, \mathrm{b} *}$

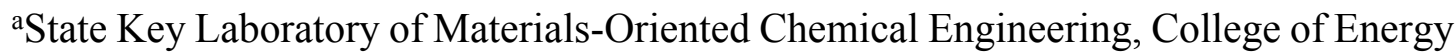

Science and Engineering and Institute of Advanced Materials, Nanjing Tech University, No.30, Puzhu Road (S), Nanjing, Jiangsu, 211800, China

bSouth China Normal University, No. 55, West Zhongshan Road, Tianhe District, Guangzhou, Guangdong, 510631, China

*Corresponding authors.

E-mail addresses: 1.fu@,njtech.edu.cn; lijunfufu@sina.com (L. Fu);

houxianhua@m.scnu.edu.cn (X.Hou); wuyp@njtech.edu.cn (Y.Wu)

\#The authors contribute equally to this work. 

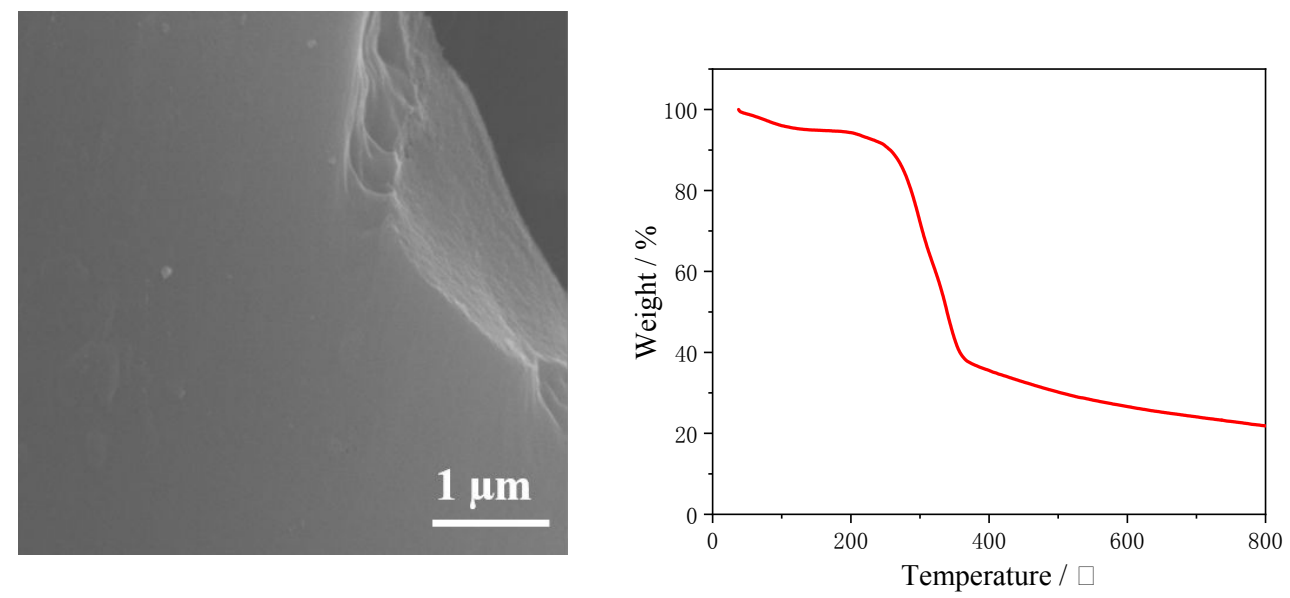

Figure S1. (A) FE-SEM image of CC sample; (B) TGA curve of corncob under inert atmosphere.

The ACC material presents high specific surface area, and leading to excellent electrochemical properties. The high specific surface area is ascribed to well controlled preparation process. The as-prepared ACC material was prepared via a precarbonization-calcination-activation approach. From the thermogravimetric analysis (TGA) curve (Figure S1B), it can be seen that the main weight loss starts at $450{ }^{\circ} \mathrm{C}$ which is ascribed to the decomposition of the organics. The pre-carbonization process would maintain the structure of corncob, at the same time form porous structure via the gas release during the decomposition process. In addition, for ACC, the subsequent activation process with $\mathrm{KOH}$ generates more porous structure. Therefore, the as-prepared ACC presents a large specific surface area. 


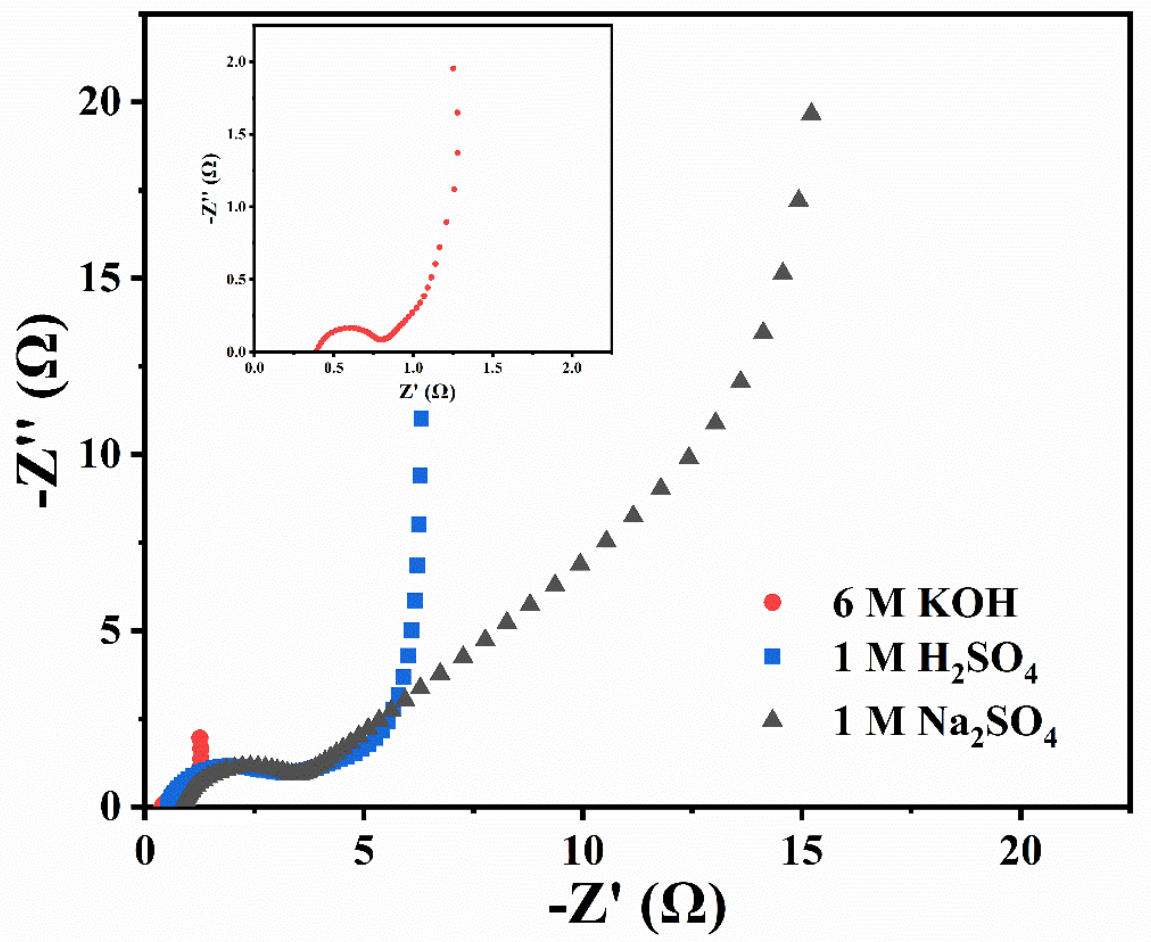

Figure S2. Nyquist plots of ACC electrode in $6 \mathrm{M} \mathrm{KOH}, 1 \mathrm{M} \mathrm{H}_{2} \mathrm{SO}_{4}$, and $1 \mathrm{M} \mathrm{Na}_{2} \mathrm{SO}_{4}$ at $\mathrm{OCV}$ before cycling (inset is Nyquist plot of ACC electrode in $6 \mathrm{M} \mathrm{KOH}$ ). 

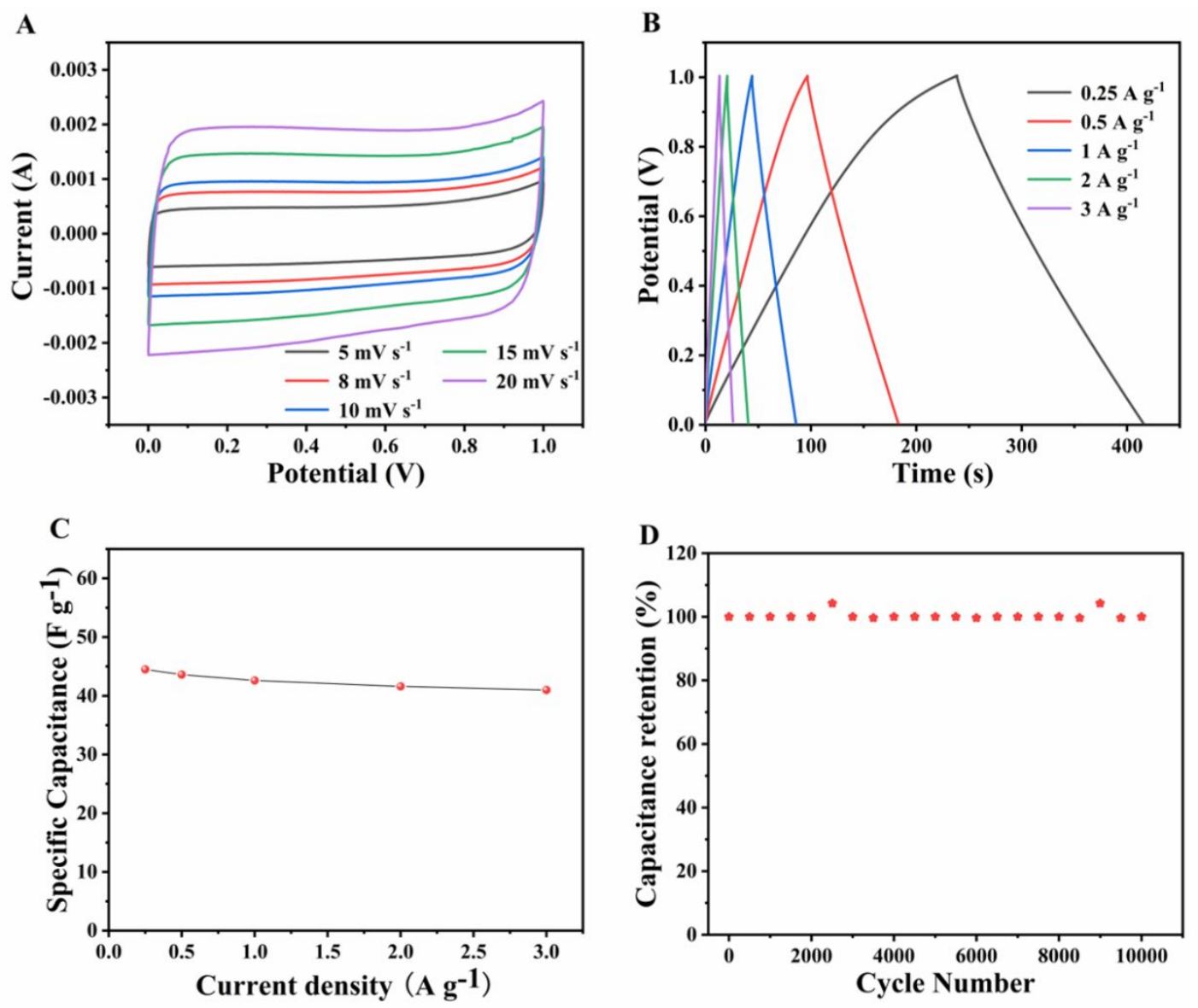

Figure S3. Electrochemical performance of the ACC based symmetric supercapacitor with two-electrode system in $6 \mathrm{M} \mathrm{KOH}$ : $\mathrm{CV}$ curves at various scan rates (A), GCD plots at different current densities (B), specific capacitance at different current densities (C), and cycling performance at $3 \mathrm{~A} \mathrm{~g}^{-1}$ over 10000 cycles (D). 

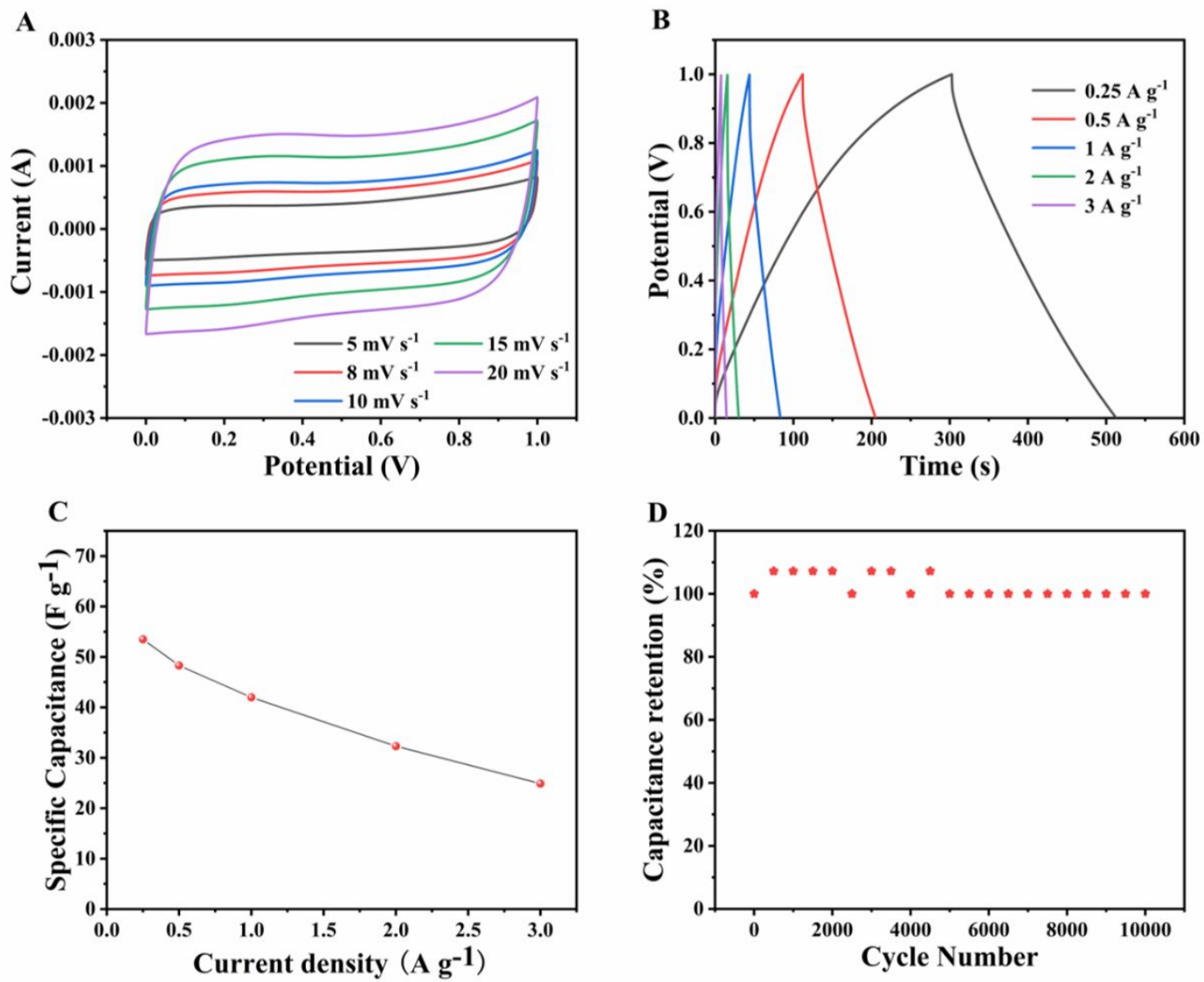

Figure S4. Electrochemical performance of the ACC based symmetric supercapacitor with two-electrode system in $1 \mathrm{M} \mathrm{H}_{2} \mathrm{SO}_{4}$ : $\mathrm{CV}$ curves at various scan rates (A), GCD plots at different current densities (B), specific capacitance at different current densities (C), and cycling performance at $3 \mathrm{~A} \mathrm{~g}^{-1}$ over 10000 cycles (D). 

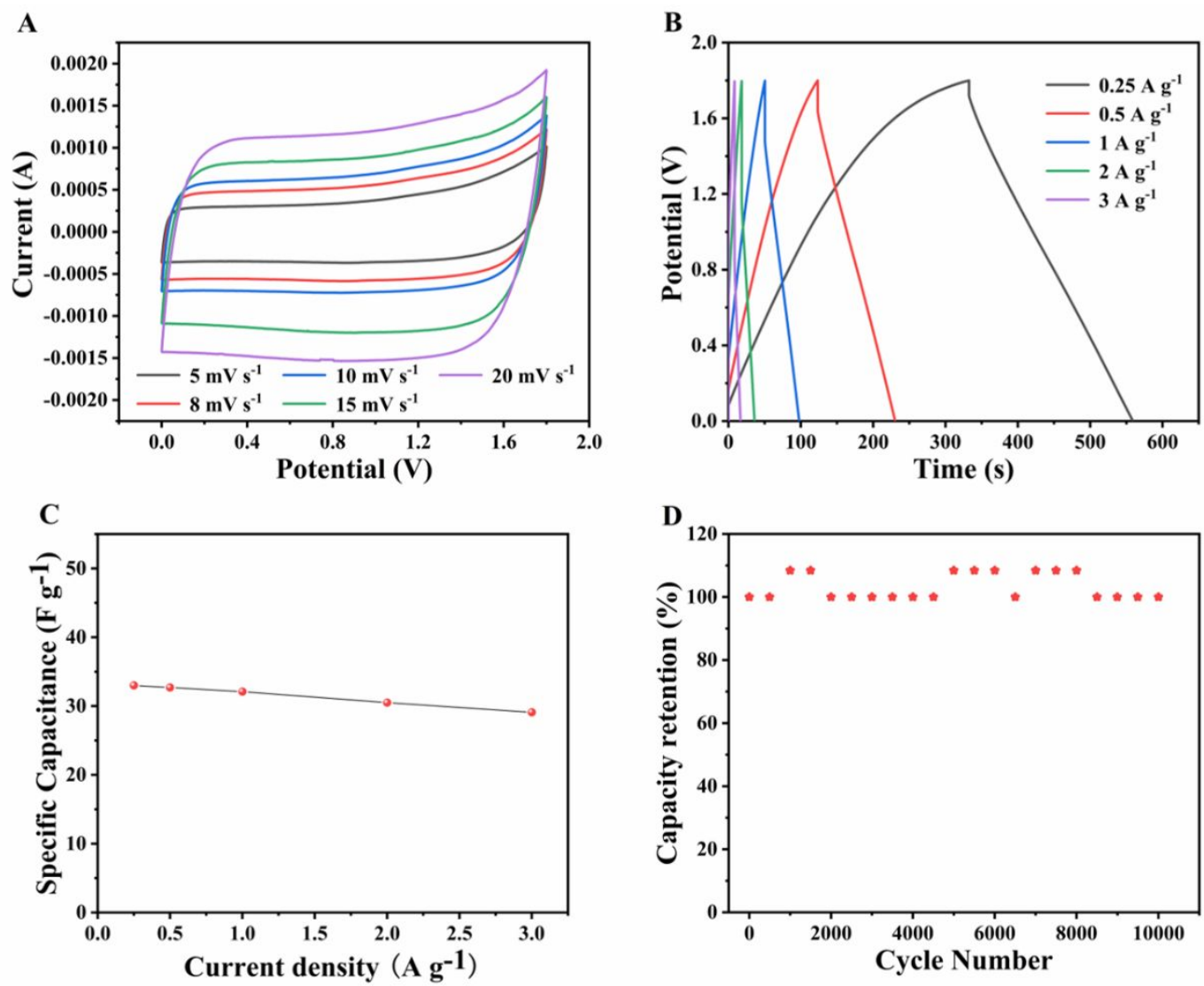

Figure S5. Electrochemical performance of the ACC based symmetric supercapacitor with two-electrode system in $1 \mathrm{M} \mathrm{Na}_{2} \mathrm{SO}_{4}$ : CV curves at various scan rates (A), GCD plots at different current densities (B), specific capacitance at different current densities (C), and cycling performance at $3 \mathrm{~A} \mathrm{~g}^{-1}$ over 10000 cycles (D). 

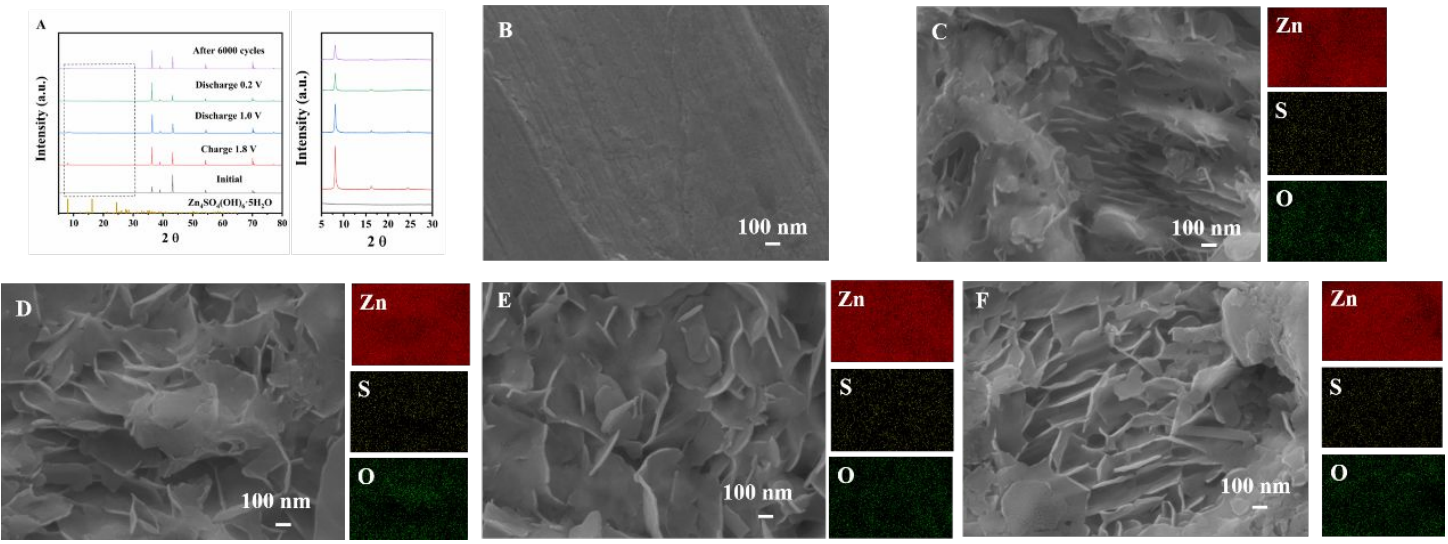

Figure S6. Ex situ XRD patterns (A), Ex situ FE-SEM and corresponding EDS mapping of the $\mathrm{Zn}$ anode at different charge / discharge states in first and $6000^{\text {th }}$ cycle in the AZHS: initial Zn foil (B), charge to $1.8 \mathrm{~V}(\mathrm{C})$, discharge to $1.0 \mathrm{~V}(\mathrm{D})$, discharge to $0.2 \mathrm{~V}(\mathrm{E})$, and full discharge after 6000 cycles $(\mathrm{F})$. 

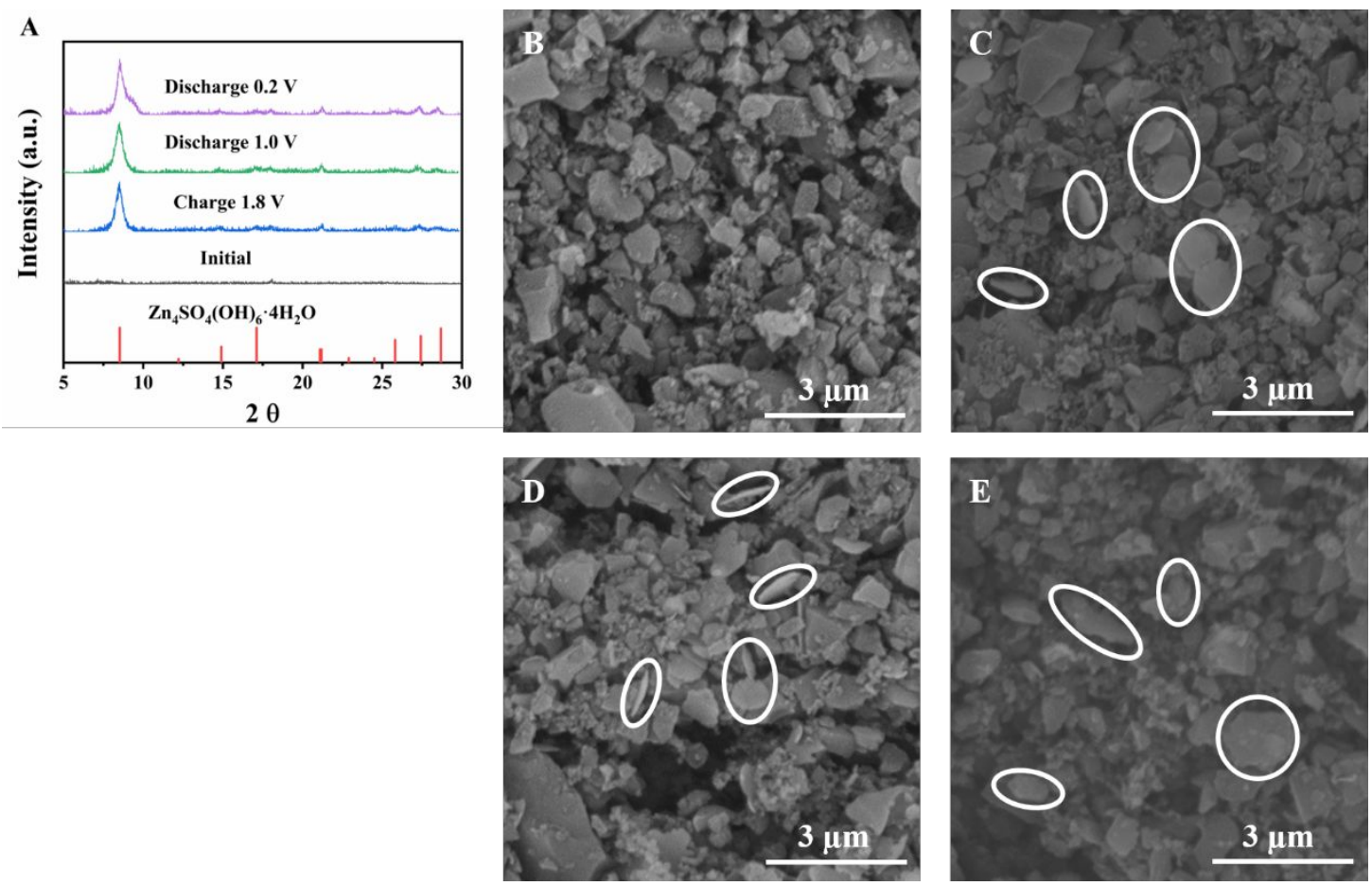

Figure S7. Ex situ XRD patterns (A), Ex situ FE-SEM and corresponding EDS mapping of the ACC cathode at different charge / discharge states in first cycle in the AZHS: initial ACC electrode (B), charge to $1.8 \mathrm{~V}(\mathrm{C})$, discharge to $1.0 \mathrm{~V}(\mathrm{D})$, and discharge to $0.2 \mathrm{~V}(\mathrm{E})$. 

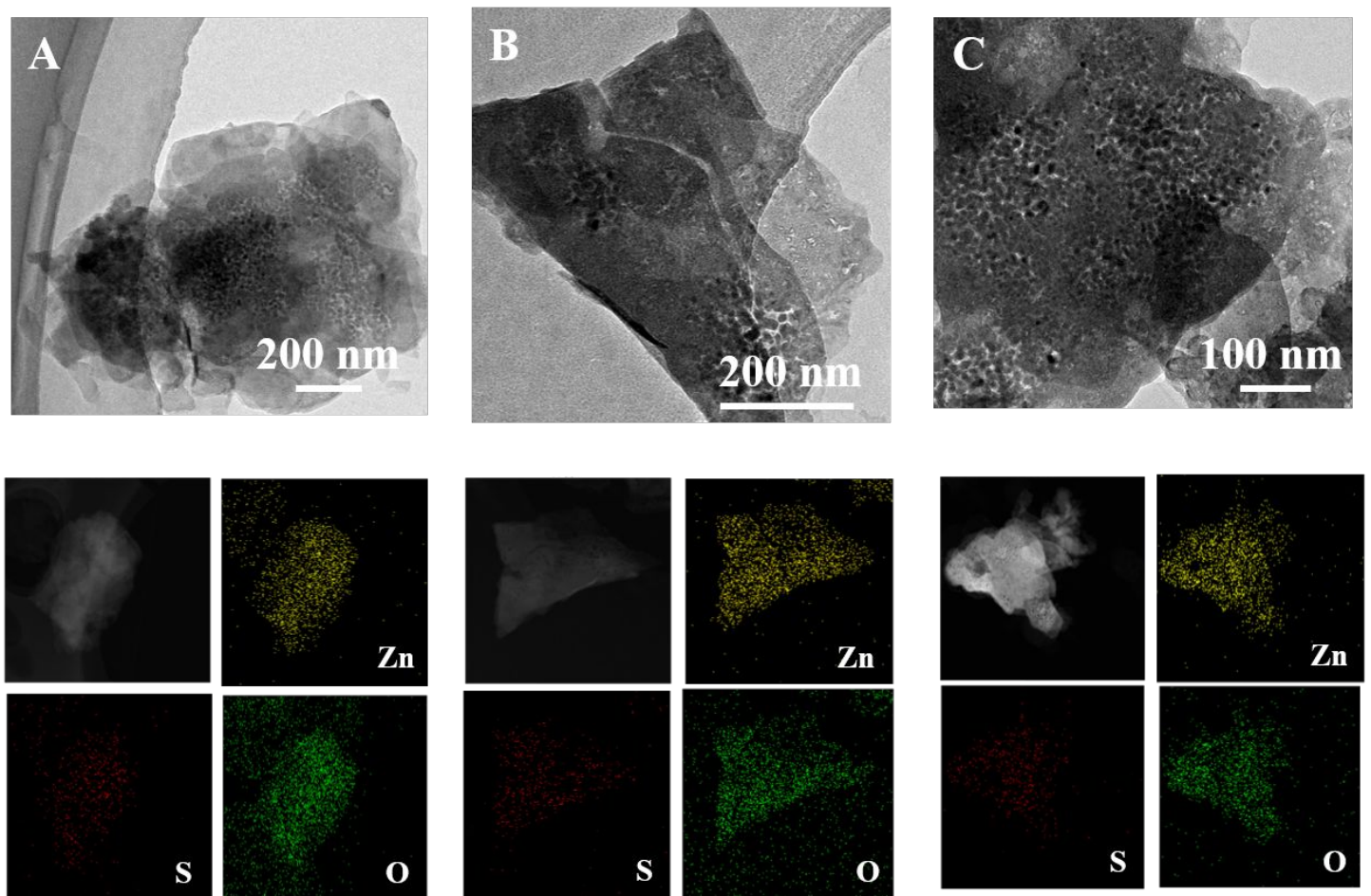

Figure S8. Ex situ TEM and HR-TEM images of the ACC cathode materials at different charge / discharge states in first cycle in the AZHS and the corresponding scanning transmission electron microscopy-energy dispersive spectroscopy (STEM-EDS) mapping of $\mathrm{Zn}, \mathrm{S}$, and $\mathrm{O}$ elements: (A), charge to $1.8 \mathrm{~V}$, (B) discharge to $1.0 \mathrm{~V}$, and (C) discharge to $0.2 \mathrm{~V}$. 
Table S1 Weight percent of elements for CC and ACC samples obtained from XPS analysis.

\begin{tabular}{cccc}
\hline \multirow{2}{*}{ Samples } & Composition (\%) & Composition (\%) & Composition (\%) \\
& $\mathrm{C}$ & $\mathrm{O}$ & $\mathrm{N}$ \\
\hline $\mathrm{CC}$ & 87.75 & 12.01 & 0.25 \\
$\mathrm{ACC}$ & 87.57 & 12.26 & 0.17 \\
\hline
\end{tabular}


Table S2 Pore structure parameters of the materials calculated from the $\mathrm{N}_{2}$ adsorptiondesorption isotherms.

\begin{tabular}{ccccccc}
\hline Samples & $\begin{array}{c}\mathbf{S}_{\text {total }} \\
\left(\mathbf{m}^{\mathbf{2}} \mathbf{g}^{-1}\right)\end{array}$ & $\begin{array}{c}\mathbf{S}_{\text {micro }} \\
\left(\mathbf{m}^{\mathbf{2}} \mathbf{g}^{-1}\right)\end{array}$ & $\begin{array}{c}\mathbf{S}_{\text {meso+macro }} \\
\left(\mathbf{m}^{\mathbf{2}} \mathbf{g}^{-1}\right)\end{array}$ & $\begin{array}{c}\mathbf{V}_{\text {total }} \\
\left(\mathbf{c m}^{\mathbf{3}} \mathbf{g}^{-1}\right)\end{array}$ & $\begin{array}{c}\mathbf{V}_{\text {micro }} \\
\left(\mathbf{c m}^{\mathbf{3}} \mathbf{g}^{-1}\right)\end{array}$ & $\begin{array}{c}\mathbf{A P S} \\
\mathbf{( n m})\end{array}$ \\
\hline CC & 600.5 & 539.3 & 61.2 & 0.269 & 0.208 & 1.793 \\
ACC & 2619 & 1335 & 1284 & 1.346 & 0.566 & 2.056 \\
\hline
\end{tabular}


Table S3 Electrochemical performances of biomass / biowaste derived activated carbon electrode materials in alkaline solution with three-electrode systems.

\begin{tabular}{|c|c|c|c|c|c|}
\hline $\begin{array}{l}\text { Precursor } \\
\text { Materials }\end{array}$ & $\begin{array}{c}\text { Specific } \\
\text { surface area } \\
\left(\mathrm{m}^{2} \mathrm{~g}^{-1}\right)\end{array}$ & Electrolyte & $\begin{array}{l}\text { Current } \\
\text { density }\end{array}$ & $\begin{array}{c}\text { Specific } \\
\text { capacitance } \\
\left(\mathrm{F} \mathrm{g} \mathrm{g}^{-1}\right)\end{array}$ & Ref. \\
\hline Bamboo & 1472 & $6 \mathrm{M} \mathrm{KOH}$ & $0.1 \mathrm{~A} \mathrm{~g}^{-1}$ & 301 & 1 \\
\hline Yogurt & 1300 & $6 \mathrm{M} \mathrm{KOH}$ & $2 \mathrm{~A} \mathrm{~g}^{-1}$ & 225 & 2 \\
\hline Fallen leaf & 1078 & $6 \mathrm{M} \mathrm{KOH}$ & $0.5 \mathrm{~A} \mathrm{~g}^{-1}$ & 310 & 3 \\
\hline Tobacco & 2115 & $6 \mathrm{M} \mathrm{KOH}$ & $0.5 \mathrm{~A} \mathrm{~g}^{-1}$ & 286 & 4 \\
\hline Willow catkin & 1533 & $6 \mathrm{M} \mathrm{KOH}$ & $0.5 \mathrm{~A} \mathrm{~g}^{-1}$ & 298 & 5 \\
\hline Wood sawdust & 2294 & $6 \mathrm{M} \mathrm{KOH}$ & $0.5 \mathrm{~A} \mathrm{~g}^{-1}$ & 225 & 6 \\
\hline Shrimp shell & 1113 & $6 \mathrm{M} \mathrm{KOH}$ & $1 \mathrm{~A} \mathrm{~g}^{-1}$ & 295 & 7 \\
\hline Bamboo & 1732 & $6 \mathrm{M} \mathrm{KOH}$ & $1 \mathrm{Ag}-1$ & 171.4 & 8 \\
\hline Lotus stem & 2194 & $6 \mathrm{M} \mathrm{KOH}$ & $5 \mathrm{mV} \mathrm{s}^{-1}$ & 174 & 9 \\
\hline Corncob sponge & 1874 & $6 \mathrm{M} \mathrm{KOH}$ & $1 \mathrm{~A} \mathrm{~g}^{-1}$ & 303.6 & 10 \\
\hline $\mathrm{ACC}$ & 2619 & $6 \mathrm{M} \mathrm{КОН}$ & $1 \mathrm{~A} \mathrm{~g}^{-1}$ & 311 & $\begin{array}{l}\text { This } \\
\text { work }\end{array}$ \\
\hline
\end{tabular}


Table S4 Electrochemical performances of biomass / biowaste derived activated carbon electrode materials in acidic solution with three-electrode systems.

\begin{tabular}{|c|c|c|c|c|c|}
\hline Materials & $\begin{array}{c}\text { Specific } \\
\text { surface area } \\
\left(\mathrm{m}^{2} \mathrm{~g}^{-1}\right)\end{array}$ & Electrolyte & $\begin{array}{l}\text { Current } \\
\text { density }\end{array}$ & $\begin{array}{c}\text { Specific } \\
\text { capacitance } \\
\left(\mathrm{F} \mathrm{g}^{-1}\right)\end{array}$ & Ref. \\
\hline Paulownia flower & 1159 & $1 \mathrm{M} \mathrm{H}_{2} \mathrm{SO}_{4}$ & $1 \mathrm{Ag}^{-1}$ & 297 & 11 \\
\hline Coconut shell & 2440 & $0.5 \mathrm{M} \mathrm{H}_{2} \mathrm{SO}_{4}$ & $0.25 \mathrm{~A} \mathrm{~g}^{-1}$ & 246 & 12 \\
\hline Defatted soybean & 2130 & $1 \mathrm{M} \mathrm{H}_{2} \mathrm{SO}_{4}$ & $0.2 \mathrm{~A} \mathrm{~g}^{-1}$ & 260 & 13 \\
\hline Cotton stalk & 1964.46 & $1 \mathrm{M} \mathrm{H}_{2} \mathrm{SO}_{4}$ & $0.2 \mathrm{~A} \mathrm{~g}^{-1}$ & 254 & 14 \\
\hline $\mathrm{ACC}$ & 2619 & $1 \mathrm{M} \mathrm{H}_{2} \mathrm{SO}_{4}$ & $1 \mathbf{A ~ g}^{-1}$ & 357 & $\begin{array}{l}\text { This } \\
\text { work }\end{array}$ \\
\hline
\end{tabular}




\section{REFERENCES}

(1) Tian, W.; Gao, Q.; Tan, Y.; Yang, K.; Zhu, L.; Yang, C.; Zhang, H., Bio-Inspired Beehive-Like Hierarchical Nanoporous Carbon Derived from Bamboo-Based Industrial By-Product as a High Performance Supercapacitor Electrode Material. Journal of Materials Chemistry A 2015, 3, 5656-5664.

(2) Wahid, M.; Parte, G.; Phase, D.; Ogale, S., Yogurt: a Novel Precursor for Heavily Nitrogen Doped Supercapacitor Carbon. Journal of Materials Chemistry A 2015, 3, 1208-1215.

(3) Li, Y.-T.; Pi, Y.-T.; Lu, L.-M.; Xu, S.-H.; Ren, T.-Z., Hierarchical Porous Active Carbon from Fallen Leaves by Synergy of $\mathrm{K}_{2} \mathrm{CO}_{3}$ and Their Supercapacitor Performance. Journal of Power Sources 2015, 299, 519-528.

(4) Zhao, Y.-Q.; Lu, M.; Tao, P.-Y.; Zhang, Y.-J.; Gong, X.-T.; Yang, Z.; Zhang, G.-

Q.; Li, H.-L., Hierarchically Porous and Heteroatom Doped Carbon Derived from Tobacco Rods for Supercapacitors. Journal of Power Sources 2016, 307, 391-400.

(5) Li, Y.; Wang, G.; Wei, T.; Fan, Z.; Yan, P., Nitrogen and Sulfur Co-Doped Porous Carbon Nanosheets Derived from Willow Catkin for Supercapacitors. Nano Energy 2016, 19, 165-175.

(6) Huang, Y.; Peng, L.; Liu, Y.; Zhao, G.; Chen, J. Y.; Yu, G., Biobased Nano Porous Active Carbon Fibers for High-Performance Supercapacitors. ACS Applied Materials \& Interfaces 2016, 8, 15205-15215. 
(7) Gao, F.; Qu, J.; Geng, C.; Shao, G.; Wu, M., Self-Templating Synthesis of NitrogenDecorated Hierarchical Porous Carbon from Shrimp Shell for Supercapacitors. Journal of Materials Chemistry A 2016, 4, 7445-7452.

(8) Gong, Y.; Li, D.; Luo, C.; Fu, Q.; Pan, C., Highly Porous Graphitic Biomass Carbon as Advanced Electrode Materials for Supercapacitors. Green Chemistry 2017, 19, $4132-4140$.

(9) Zhang, Y.; Liu, S.; Zheng, X.; Wang, X.; Xu, Y.; Tang, H.; Kang, F.; Yang, Q.-H.; Luo, J., Biomass Organs Control the Porosity of Their Pyrolyzed Carbon. Advanced Functional Materials 2017, 27, 1604687.

(10) Liu, Y.; Xiao, Z.; Liu, Y.; Fan, L.-Z., Biowaste-Derived 3D Honeycomb-Like Porous Carbon with Binary-Heteroatom Doping for High-Performance Flexible SolidState Supercapacitors. Journal of Materials Chemistry A 2018, 6, 160-166.

(11) Chang, J.; Gao, Z.; Wang, X.; Wu, D.; Xu, F.; Wang, X.; Guo, Y.; Jiang, K., Activated Porous Carbon Prepared from Paulownia Flower for High Performance Supercapacitor Electrodes. Electrochimica Acta 2015, 157, 290-298.

(12) Jain, A.; Xu, C.; Jayaraman, S.; Balasubramanian, R.; Lee, J. Y.; Srinivasan, M. P., Mesoporous Activated Carbons with Enhanced Porosity by Optimal Hydrothermal Pre-Treatment of Biomass for Supercapacitor Applications. Microporous and Mesoporous Materials 2015, 218, 55-61.

(13) Ferrero, G. A.; Fuertes, A. B.; Sevilla, M., From Soybean Residue to Advanced Supercapacitors. Scientific Reports 2015, 5, 16618. 
(14) Tian, X.; Ma, H.; Li, Z.; Yan, S.; Ma, L.; Yu, F.; Wang, G.; Guo, X.; Ma, Y.;

Wong, C., Flute Type Micropores Activated Carbon from Cotton Stalk for High Performance Supercapacitors. Journal of Power Sources 2017, 359, 88-96. 